\title{
Large-eddy simulation of the compressible flow past a tabbed cylinder
}

\author{
XU ChangYue*, ZHAO LiQing \& SUN JianHong \\ College of Aerospace Engineering, Nanjing University of Aeronautics \& Astronautics, Nanjing 210016, China
}

Received December 19, 2011; accepted March 1, 2012

\begin{abstract}
The compressible flow past a tabbed cylinder has been studied numerically using large-eddy simulation for a free-stream Mach number $M_{\infty}=0.75$ and a Reynolds number based on the diameter $R e=2 \times 10^{5}$. Because of the passive control of the flow past a tabbed cylinder, the mean drag coefficient of tabbed cylinder is less than that of a corresponding circular cylinder with a drag reduction up to $33 \%$. The fluctuating lift coefficient is greatly suppressed to be nearly zero. Drag reduction due to the shearing process prevails over that due to the compressing process in this flow. Through investigating the mechanisms relevant to passive control of the tab, it is found that suppression of the shear layer instability can lead to a higher-base-pressure distribution, which can reasonably be associated with drag reduction and suppression of the lift fluctuations. The analysis of convective Mach number and self-sustained oscillations phenomenon inside the shear layers indicates that both the compressible effect and high-frequency forcing result in suppression of the shear layer instability of tabbed cylinder.
\end{abstract}

circular cylinder, shock wave, compressible turbulence, large-eddy simulation

Citation: Xu C Y, Zhao L Q, Sun J H. Large-eddy simulation of the compressible flow past a tabbed cylinder. Chin Sci Bull, 2012, 57: 3203-3210, doi: 10.1007/ s11434-012-5313-X

Researches on the control of flow past a bluff body are of interest in the fundamental and engineering problems, typically a canonical geometry circular cylinder. Recently, a review on the control of flow past a bluff body has been given by Choi et al. [1]. Control methods for bluff body have been classified as boundary-layer controls and directwake modifications, depending on whether the control delays separation by modifying the boundary layer or changes directly the wake characteristics. In the direct-wake control methods, two groups were classified in terms of twodimensional (2D) or three-dimensional (3D) disturbances in the spanwise direction of bluff body [1]. One group was called "2D forcing", and the other group was named "3D forcing".

By means of the "2D forcing" direct-wake control, some works have been performed for flow past a circular cylinder with splitter plates or tabs [2-5]. Anderson et al. [2] used hot wire to measure effects of a splitter plate on the near

*Corresponding author (email: cyxu@nuaa.edu.cn) wake of a circular cylinder. Hwang et al. [3] investigated numerically the optimal location of the plate for maximum reduction. Akilli et al. [4] performed experiments to study effect of the splitter plate length on the flow characteristics. Thombi et al. [5] studied experimentally and numerically the flow control on a circular cylinder at transonic speed by inserting tabs inside the separated region. These studies reveal that the splitter plate or tab can lead to the formation of an elongated dead water region, which can yield a higher base pressure. Many investigations have been found to deal with the turbulent flow over a circular cylinder with splitter plate. However, it is necessary to do more detail work in order to understand the essential flow control mechanisms of the tab especially in the transonic region.

The transonic flow past a circular cylinder, which is a prototype of tabbed cylinder, is introduced to understand its underlying complex flow phenomena, such as, shock-wave and turbulent-boundary-layer interaction, shock-wave and wake interaction, and compressible shear layer instability. Experiments of the transonic flow past a circular cylinder 
have been performed by Macha [6], Murthy and Rose [7], and Rodriguez [8] for the Reynolds number about $10^{5}$. The 2D compressible Navier-Stokes equations are numerically solved using a finite volume method to investigate the unsteady forces and flow structures near the cylinder at $M_{\infty}=0.8$ [9]. Effect of Mach number on transonic flow past a circular cylinder has been studied numerically [10]. Recently, passive control of the compressible flow past a wavy cylinder has been carried out using large-eddy simulation [11]. Compared with the incompressible flow control, some phenomena relevant to the compressible effect have been analyzed.

In this paper, a large-eddy simulation (LES) technique, which has provided a powerful tool for studying the dynamics of turbulent flows, is utilized to investigate the compressible flow past a tabbed cylinder and a corresponding circular cylinder. The purpose is to achieve an improved understanding of the passive control mechanisms of the tabs.

\section{Mathematical formulation and method}

\subsection{Governing equations}

Large eddy simulations are implemented in the present work for turbulence closure, in which large-scale motions are explicitly calculated and eddies with scales smaller than the grid or filter size are modeled to represent the effects of unresolved motions on resolved scales. The 3D Favrefiltered conservation equations of mass, momentum, and energy are employed, which can be expressed in the following conservative form [11]:

$$
\begin{aligned}
& \frac{\partial \bar{\rho}}{\partial t}+\frac{\partial\left(\bar{\rho} \tilde{u}_{i}\right)}{\partial x_{i}}=0, \\
& \frac{\partial\left(\bar{\rho} \tilde{u}_{i}\right)}{\partial t}+\frac{\partial\left(\bar{\rho} \tilde{u}_{i} \tilde{u}_{j}\right)}{\partial x_{j}}=-\frac{\partial \bar{p}}{\partial x_{i}}+\frac{\partial\left(\tilde{\tau}_{i j}-\tau_{i j}^{\mathrm{SGS}}+D_{i j}^{\mathrm{SGS}}\right)}{\partial x_{j}}, \\
& \frac{\partial(\bar{\rho} \tilde{E})}{\partial t}+\frac{\partial\left[(\bar{\rho} \tilde{E}+\bar{p}) \tilde{u}_{i}\right]}{\partial x_{i}}=\frac{\partial}{\partial x_{i}}\left(-\tilde{q}_{i}+\tilde{u}_{j} \tilde{\tau}_{i j}+J_{i}^{\mathrm{SGS}}\right. \\
& \left.+\sigma_{i}^{\mathrm{SGS}}-Q_{i}^{\mathrm{SGS}}-H_{i}^{\mathrm{SGS}}\right) \text {, }
\end{aligned}
$$

where an overbar denotes the spatial filter and a tilde represents the Favre filter, i.e. $\tilde{f}=\overline{\rho f} / \bar{\rho}$. The variables $\rho$, $u_{i}, p$, and $E$ represent the density, velocity component, pressure and specific total energy, respectively. The diffusive fluxes are given by

$$
\begin{gathered}
\tilde{\tau}_{i j}=2 \tilde{\mu} \tilde{S}_{i j}-\frac{2}{3} \tilde{\mu} \delta_{i j} \tilde{S}_{k k}, \\
\tilde{q}_{i}=-\frac{\tilde{\mu} C_{p}}{\operatorname{Pr}} \frac{\partial \tilde{T}}{\partial x_{i}},
\end{gathered}
$$

where $\mu$ is the molecular viscosity, $C_{p}$ is the constant-pressure specific heat, $\mathrm{Pr}$ is the Prandtl number and
$S_{i j} \quad$ represents the strain-rate tensor defined as $S_{i j}=\left(\partial u_{i} / \partial x_{j}+\partial u_{j} / \partial x_{i}\right) / 2$. The perfect gas relationship and Sutherland law for the molecular viscosity coefficient $\mu$ are employed. Subgrid closure terms in eqs. (2) and (3) are defined as

$$
\begin{gathered}
\tau_{i j}^{\mathrm{SGS}}=\bar{\rho}\left(\widetilde{u_{i} u_{j}}-\tilde{u}_{i} \tilde{u}_{j}\right), \\
D_{i j}^{\mathrm{SGS}}=\bar{\tau}_{i j}-\tilde{\tau}_{i j}, \\
J_{i}^{\mathrm{SGS}}=-\frac{1}{2} \bar{\rho}\left(\widetilde{u_{i} u_{k} u_{k}}-\widetilde{u_{i}} \widetilde{u_{k} u_{k}}\right), \\
Q_{i}^{\mathrm{SGS}}=\bar{q}_{i}-\tilde{q}_{i}, \\
H_{i}^{\mathrm{SGS}}=C_{p}\left(\widetilde{\rho} \widetilde{u_{i} T}-\bar{\rho} \tilde{u}_{i} \tilde{T}\right), \\
\sigma_{i}^{\mathrm{SGS}}=\overline{u_{j} \tau_{i j}}-\tilde{u}_{j} \tilde{\tau}_{i j} .
\end{gathered}
$$

These terms arise from the unresolved scales and need to be modeled in terms of the resolved scales, which will be described in the following section.

\subsection{Subgrid-scale models}

The introduction of the dynamic model proposed by Germano et al. [12] has spurred significant progress in the subgrid-scale (SGS) modeling of non-equilibrium flows. Among the various SGS turbulence models, such as the mixed model [13] and the two-parameter mixed model [14], the dynamic SGS model for turbulence closure offers the best trade-off between accuracy and cost [15]. In the present study, the dynamic Smagorinsky model for compressible flows proposed by Moin et al. [16] is employed. The anisotropic part of the SGS stresses (6) is treated using the Smagorinsky model [17], while the isotropic part $\tau_{k k}^{\mathrm{SGS}}$ is modeled with a formulation proposed by Yoshizawa [18]:

$$
\begin{aligned}
\tau_{i j}^{\mathrm{SGS}}-\frac{1}{3} \delta_{i j} \tau_{k k}^{\mathrm{SGS}} & =-2 C_{R} \bar{\Delta}^{2} \bar{\rho}|\tilde{S}|\left(\tilde{S}_{i j}-\frac{1}{3} \delta_{i j} \tilde{S}_{k k}\right) \\
& =C_{R} \alpha_{i j}, \\
\tau_{k k}^{\mathrm{SGS}} & =2 C_{I} \bar{\Delta}^{2} \bar{\rho}|\tilde{S}|^{2}=C_{I} \alpha .
\end{aligned}
$$

The subgrid energy flux term is

$$
H_{i}^{\mathrm{SGS}}=C_{p}\left(\bar{\rho} \widetilde{u_{i} T}-\bar{\rho} \tilde{u}_{i} \tilde{T}\right)=-\frac{\tilde{\mu}_{t} C_{p}}{P r_{t}} \frac{\partial \tilde{T}}{\partial x_{j}} .
$$

The SGS turbulent diffusion terms is reasonably modeled as $J_{i}^{\mathrm{SGS}}=\tilde{u}_{j} \tau_{i j}^{\mathrm{SGS}}$ [19]. Treatments of the viscous stress term, $D_{i j}^{\mathrm{SGS}}$, and the heat flux term, $Q_{i}^{\mathrm{SGS}}$, have been analyzed by Piomelli [20]. The SGS viscous diffusion term $\sigma_{i}^{\mathrm{SGS}}$ is neglected because of its small contribution in the energy equation [21]. The model coefficients $C_{R}$ and $C_{I}$ are determined dynamically during the calculation. A detailed description of the dynamics SGS model can be found in our previous paper [11]. 


\subsection{Numerical procedure}

Governing equations are numerically solved by the finitevolume method. The temporal integration is performed using an implicit approximate-factorization method with sub-iterations to ensure the second-order accuracy. Both the convective and diffusive terms are discretized with second-order central schemes, and a fourth-order low artificial numerical dissipation is employed to prevent numerical oscillations at high wavenumbers [11]. To capture the discontinuity caused by shock wave, a second-order upwind scheme with the Roe's flux-difference splitting is introduced into the inviscid flux. The artificial dissipation is also turned off in the region in which the upwind scheme works. A binary sensor function $\Phi_{i+1 / 2}$ at cell face $i+1 / 2$ is used for the detection of shock waves; $\Phi_{i+1 / 2}$ is determined by the pressure and density curvature criteria proposed by Hill et al. [22]:

$$
\alpha_{p}^{i+1 / 2}=\max \left(\alpha_{p}^{i+1}, \alpha_{p}^{i+2}\right), \alpha_{\rho}^{i+1 / 2}=\max \left(\alpha_{\rho}^{i+1}, \alpha_{\rho}^{i+2}\right),
$$

and

$$
\alpha_{p}^{i}=\left|\frac{p_{i+1}-2 p_{i}+p_{i-1}}{p_{i+1}+2 p_{i}+p_{i-1}}\right|, \alpha_{\rho}^{i}=\left|\frac{\rho_{i+1}-2 \rho_{i}+\rho_{i-1}}{\rho_{i+1}+2 \rho_{i}+\rho_{i-1}}\right|,
$$

where $\alpha_{p}^{i}$ and $\alpha_{\rho}^{i}$ represent the pressure and density relative curvatures at cell centre, respectively. When $\alpha_{p}^{i+1 / 2}>c_{1}$ and $\alpha_{\rho}^{i+1 / 2}>c_{2}, \Phi_{i+1 / 2}$ is 1 ; but zero, otherwise. The $3 \mathrm{D}$ version of this detection is used in the simulations. Similar to the treatment [22], the value of $c_{1}$ and $c_{2}$ that proved to give the best results are chosen as 0.01 [11]. Based on this detection, the Roe's second-order upwind flux only operates at the cells in the vicinity of shock waves.

In this study, the initial condition is set as the free-stream quantities. The far field boundary conditions are treated by local one-dimensional Riemann-invariants. No-slip and adiabatic conditions are applied on the cylinder and tab surface. Periodic condition is used in the axial direction of the cylinder.

\section{Results and discussion}

\subsection{Simulation overview}

Two symmetrical tabs are installed on the cylinder surface downstream of the time-averaged separation point, as shown in Figure 1. Corresponding to the experimental measurement [5], these tabs are located at $\theta_{t}=130^{\circ}$ with length $h / D=0.2$. Here, $D$ represents diameter of the cylinder, and $h$ is the height of tabs. In fact, it is the case of circular cylinder when $h=0$. Based on some typical experiments for the compressible flow past a circular cylinder [6-8] and tabbed cylinder [5], we choose the free-stream Mach number $M_{\infty}$ as 0.75 and Reynolds number based on the diameter of cylinder $R e$ as $2 \times 10^{5}$. Grids are of O-type with a

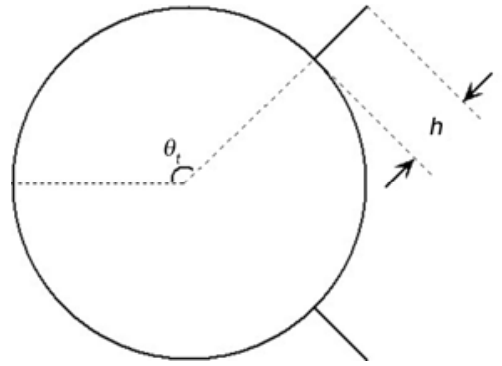

Figure 1 Schematic diagram of a tabbed cylinder.

far-field boundary at $50 \mathrm{D}$ away from the cylinder in the cross-section, and spanwise length $4 D$. After our careful tests [11], grid number is $513 \times 513 \times 121$ in the radial, azimuthal, and spanwise direction, respectively, and time step $0.002 \mathrm{D} / U_{\infty}$. For improving computational efficiency, the present code is equipped with a multi-block domain decomposition feature to facilitate parallel processing in a distributed computing environment.

Based on the time-dependent resolved density, $\bar{\rho}$, pressure, $\bar{p}$, temperature, $\tilde{T}$ and velocity, $\tilde{u}_{i}$, several averaging operations will be needed. To clearly present the post-process, some symbols used in this paper are introduced as follows: \langle\rangle means the average in time as well as in spanwise direction, and $\{\phi\}=\langle\bar{\rho} \phi\rangle /\langle\bar{\rho}\rangle$ with a variable $\phi$. Then, their fluctuations are obtained as $\rho^{\prime}=\bar{\rho}-\langle\bar{\rho}\rangle$, $p^{\prime}=\bar{p}-\langle\bar{p}\rangle, T^{\prime \prime}=\tilde{T}-\{\tilde{T}\}$ and $u_{i}^{\prime \prime}=\tilde{u}_{i}-\left\{\tilde{u}_{i}\right\}$, respectively.

\subsection{Force behaviors}

The behavior of the forces exerting on the cylinder is an important issue related to the control of flow over a bluff body [1]. Figure 2 shows the time-dependent lift and drag coefficients $C_{L}$ and $C_{D}$ on the circular and tabbed cylinder. It is seen that obvious reductions of mean drag and fluctuating lift on the tabbed cylinder occur with respect to those on the circular cylinder. To obtain the reductions of drag and fluctuating lift quantitatively, Table 1 shows timeaveraged drag coefficient $\left\langle C_{D}\right\rangle_{t}$ and root-mean-square value of lift coefficient fluctuation $C_{L r m s}$ of the two cylinders. We firstly compare with the values of $\left\langle C_{D}\right\rangle_{t}$ on both the cylinders and notice that $\left\langle C_{D}\right\rangle_{t}$ of tabbed cylinder is less than that of circular cylinder with a drag reduction up to $33 \%$. Then, comparing with the values of $C_{L \mathrm{rms}}$, it is reasonably identified that the force fluctuation on tabbed cylinder is significantly suppressed. Moreover, validation of the computational results listed in Table 1 is also performed. It is exhibited that the present results agree well with the experimental data. 

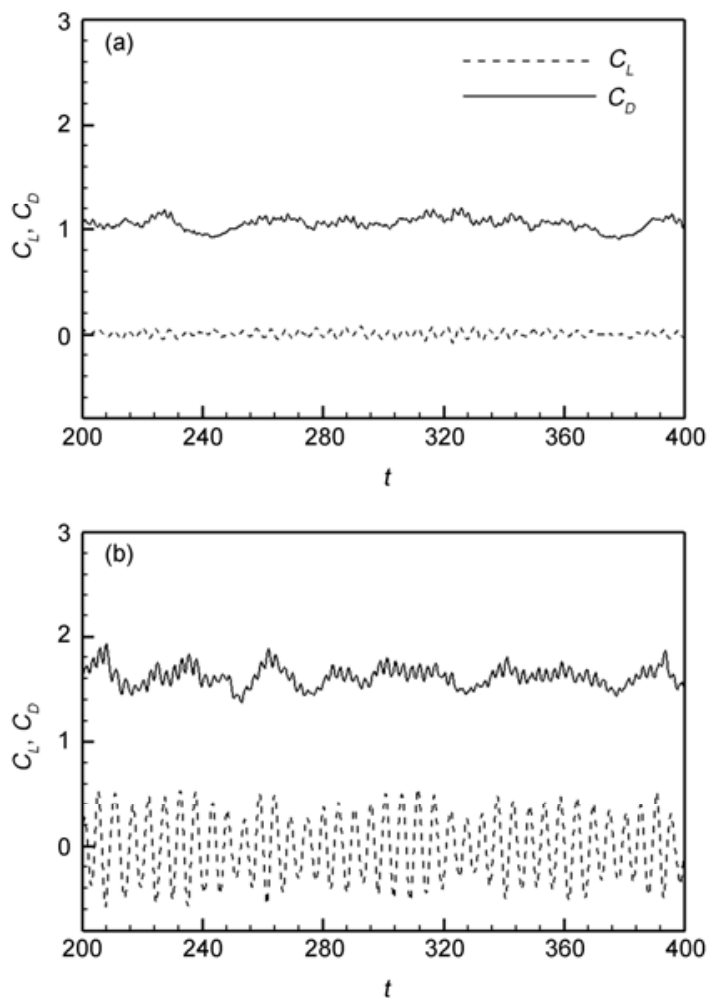

Figure 2 Time-dependent lift and drag coefficients on the tabbed cylinder (a) and circular cylinder (b).

Table 1 Comparison of the present calculated results with experimental data ${ }^{\text {a) }}$

\begin{tabular}{ccccccc}
\hline & Tabbed & Circular & EXP-1 & EXP-2 & EXP-3 & EXP-4 \\
\hline$\left\langle C_{D}\right\rangle_{t}$ & 1.055 & 1.568 & 1.45 & 1.618 & 1.5 & $1.5-1.6$ \\
$C_{L \mathrm{rms}}$ & 0.036 & 0.255 & - & 0.253 & - & -
\end{tabular}

a) Here, EXP-1 for tabbed cylinder from Thombi et al. [5] for $M_{\infty}=0.73$ and $R e=4.8 \times 10^{5}$. EXP-2, 3 and 4 represent respectively the experimental data for circular cylinder from Rodriguez [8] for $M_{\infty}=0.75$ and $R e=1.7 \times 10^{5}-3.4 \times 10^{5}$, from Murthy and Rose [7] for $M_{\infty}=0.8$ and $R e=1.66 \times 10^{5}$, and from Macha [6] for $M_{\infty}=0.8$ and $R e=10^{5}-10^{6}$

Usually, the force fluctuation on a cylinder is associated with the vortex shedding in the wake. To identify the frequency of vortex shedding, power spectral densities of time-dependent lift coefficient of the tabbed and circular cylinder are shown in Figure 3. The characteristic Strouhal number is defined as $S t=f D / U_{\infty}$ with $f$ being the frequency. As exhibited in Figure 3 for the circular cylinder, the primary frequency corresponding to the highest peak is around 0.19 , consistent with experimental data $[7,8]$ in the range $0.18-0.2$. The spectral peaks for the tabbed cylinder are relatively weak, indicating an obvious suppression of the flow unsteadiness.

The forces exerted on a body for viscous compressible flow around it depend strongly on the dynamic processes [11], such as shearing and compressing ones. To understand

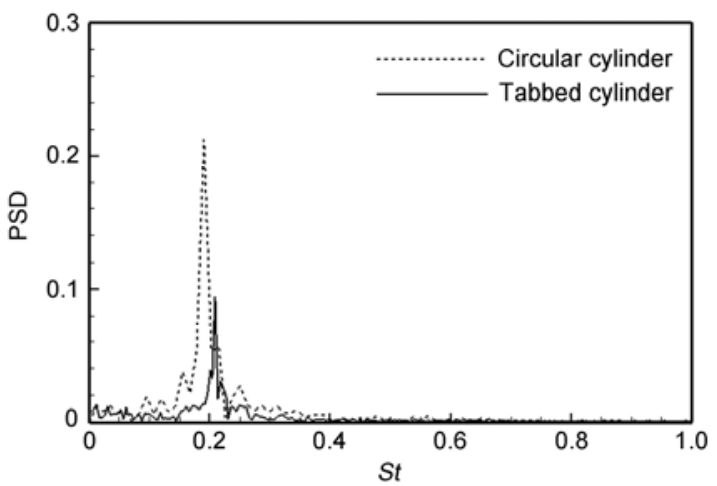

Figure 3 Profiles of power spectral density (PSD) of time-dependent lift coefficient for the tabbed and circular cylinder.

the mechanisms underlying the compressible flow past the cylinders, the force expression based on derivative-moment transformations $[23,24]$ is used to analyze the drag-force contributions. Let $V_{f}$ be a 3D compressible fluid domain surrounding a rigid and stationary body, $B$, and bounded externally by an arbitrary control surface, $\sum$, then the force formulation in terms of the resolved variables is expressed as [23]

$$
\begin{aligned}
\boldsymbol{F}= & \boldsymbol{F}_{\Sigma}+\boldsymbol{F}_{A}-\frac{1}{2} \int_{V_{f}} \boldsymbol{x} \times \nabla \times\left(\bar{\rho} \frac{\partial \tilde{\boldsymbol{u}}}{\partial t}\right) \mathrm{d} V \\
& -\int_{V_{f}}\left(\bar{\rho} \tilde{\boldsymbol{\omega}} \times \tilde{\boldsymbol{u}}-\frac{1}{2}(\tilde{\boldsymbol{u}} \cdot \tilde{\boldsymbol{u}}) \nabla \bar{\rho}\right) \mathrm{d} V \\
& -\frac{1}{2} \int_{\partial V_{f}} \boldsymbol{x} \times\left[\boldsymbol{n} \times\left(\bar{\rho} \tilde{\boldsymbol{\omega}} \times \tilde{\boldsymbol{u}}-\frac{1}{2}(\tilde{\boldsymbol{u}} \cdot \tilde{\boldsymbol{u}}) \nabla \bar{\rho}\right)\right] \mathrm{d} S,
\end{aligned}
$$

where $\tilde{\boldsymbol{\omega}}=\nabla \times \tilde{\boldsymbol{u}}$ is the vorticity, $\boldsymbol{x}$ denotes the spatial vector and $\partial V_{f}=\partial B+\Sigma$ is the surrounding boundary of $V_{f}$ with $\partial B$ being the body surface. The boundary integral in (17)

$$
\boldsymbol{F}_{\Sigma}=-\frac{1}{2} \int_{\Sigma} \boldsymbol{x} \times[\boldsymbol{n} \times(\nabla \times \tilde{\mu} \tilde{\boldsymbol{\omega}})] \mathrm{d} S+\int_{\Sigma} \tilde{\mu} \tilde{\boldsymbol{\omega}} \times \boldsymbol{n} \mathrm{d} S
$$

is the contribution to the force of flow structures on the finite control surface $\Sigma$, which is a pure viscous effect.

We imposed a periodic condition in the spanwise direction in the present calculation, so that only a finite length of the cylinder lies in the computational domain. $V_{f}$ is a doubly connected domain, and both the control surface and body surface are open surfaces. Then an additional term due to the open control surface in (17) is expressed as [23]

$$
\boldsymbol{F}_{A}=\frac{1}{2} \int_{\partial B} \boldsymbol{x} \times(\bar{p} \mathrm{~d} \boldsymbol{x}-2 \tilde{\mu} \tilde{\boldsymbol{\omega}} \times \mathrm{d} \boldsymbol{x})+2 \int_{\partial B} \tilde{\mu} \tilde{\boldsymbol{u}} \times \mathrm{d} \boldsymbol{x} .
$$

As a check, when $\Sigma$ shrinks to coincide with $\partial B$ so that all volume integrals over $V_{f}$ disappear, eq. (17) degenerates to the boundary vorticity flux based force expression for an open body surface [24].

According to the analysis of force decomposition [23], 
the terms in eq. (17), i.e. $-(\tilde{\boldsymbol{u}} \cdot \tilde{\boldsymbol{u}}) \nabla \bar{\rho} / 2$ and $\bar{\rho} \tilde{\boldsymbol{\omega}} \times \tilde{\boldsymbol{u}}$, are related to the compressing effect and vortex force, respectively. Then, the force components corresponding to the two terms are given as

$$
\begin{gathered}
\boldsymbol{F}_{C}=\frac{1}{2} \int_{\partial V_{f}} \boldsymbol{x} \times \boldsymbol{n} \times\left(\frac{1}{2}(\tilde{\boldsymbol{u}} \cdot \tilde{\boldsymbol{u}}) \nabla \bar{\rho}\right) \mathrm{d} S \\
+\frac{1}{2} \int_{V_{f}}(\tilde{\boldsymbol{u}} \cdot \tilde{\boldsymbol{u}}) \nabla \bar{\rho} \mathrm{d} V, \\
\boldsymbol{F}_{V}=-\int_{V_{f}}(\bar{\rho} \tilde{\boldsymbol{\omega}} \times \tilde{\boldsymbol{u}}) \mathrm{d} V-\frac{1}{2} \int_{\partial V_{f}} \boldsymbol{x} \times[\boldsymbol{n} \times(\bar{\rho} \tilde{\boldsymbol{\omega}} \times \tilde{\boldsymbol{u}})] \mathrm{d} S .
\end{gathered}
$$

Here we use eq. (17) to analyze the drag reduction because of the compressing and shearing process. The boundary integral (18) is calculated. Since this term is a pure viscous effect, its value is negligibly small when the control surface $\Sigma$ is chosen as over $40 D$ away from the cylinder in the cross-section plane, which is used to calculate other terms in eq. (17). The term due to the open control surface (19) contributes approximately half the total drag force for high-Reynolds-number flow, consistent with the result for viscous flow around a circular cylinder [24].

Further, we mainly discuss the drag contributions related to the compressing effect and the vortex force. The mean values of drag coefficient using eq. (20) are obtained as approximately 0.32 and 0.45 for the tabbed cylinder and circular cylinder, respectively; the drag reduction due to the compressing effect is around $29 \%$. Similarly, the drag coefficient values due to the vortex force (21) are 0.23 and 0.34 for the tabbed cylinder and circular cylinder, and the corresponding drag reduction is approximately $32 \%$. Then, we may learn that the drag reduction due to the shearing process prevails over that due to the compressing process in this flow.

The forces on the cylinder are contributed by the pressure and viscous shear stress, respectively. As the Reynolds number considered here is $O\left(10^{5}\right)$, the pressure force plays a dominant role in the total forces on the cylinder. To understand their relations to the reduction of mean and fluctuating forces on the tabbed cylinder, Figure 4 shows the mean pressure and its fluctuation, which indicates that the base pressure force of tabbed cylinder is divided into two parts by the tab. Compared with the circular cylinder, the base pressure force downstream the tab is higher, but lower upstream the tab. From the control perspective, to increase base pressure, the wake instability or shear layer instability must be suppressed. Some details about the shear layer instability will be discussed in the following section. Figure 4(b) shows that the pressure fluctuation $p_{\text {rms }}^{\prime}$ obviously exists in the base region of the cylinder. It is identified that the pressure fluctuation on the tabbed cylinder is significantly suppressed in the base region, resulting in a reduction of $C_{L \mathrm{rms}}$ on tabbed cylinder listed in Table 1. Furthermore, the calculated wall pressure coefficient and pressure fluctu
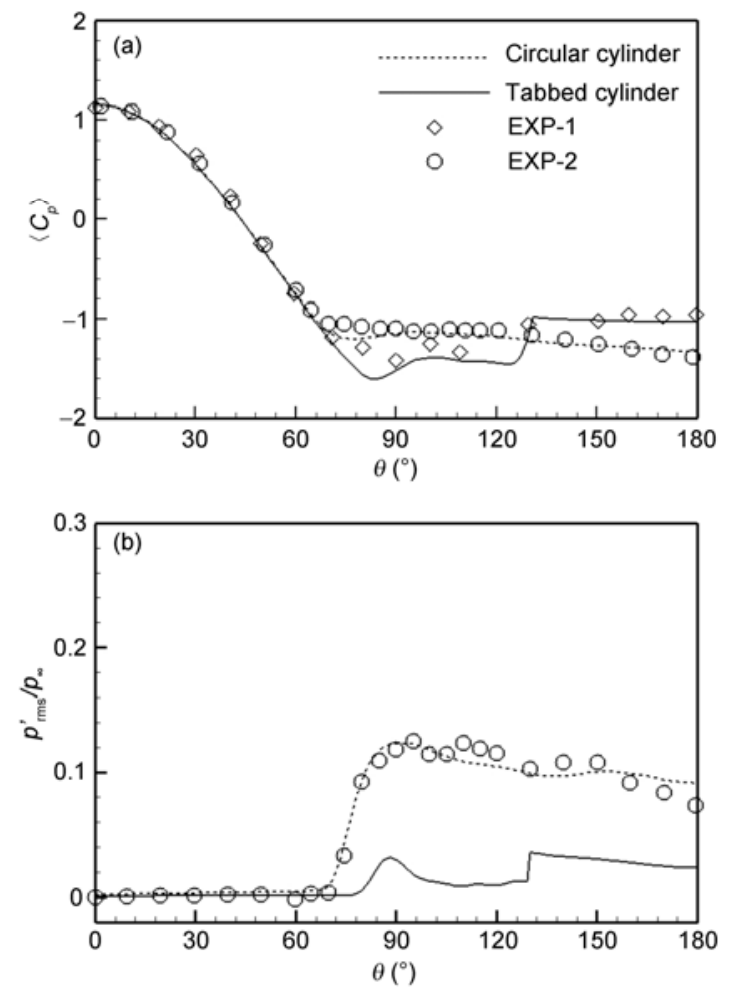

Figure 4 Comparison of the present calculated results with experimental data. (a) Mean wall pressure coefficient with the experimental data for tabbed cylinder [5] (EXP-1) and circular cylinder [8] (EXP-2); (b) root-mean-square (rms) value of pressure fluctuation with the experimental data for circular cylinder [8].

ation show good agreements with the experimental measurements, which indicates that the present results are reliable.

\subsection{Self-sustained oscillations along the shear layer}

For flow control of the bluff body, drag reduction is closely associated with the shear layer instability [25]. To analyze the flow control mechanism relevant to the shear layer instability, Figure 5 shows the instantaneous iso-contours of local Mach number $M_{l}$. It reveals the existence of two oblique shock waves in the flow field of tabbed cylinder, with one formed at the separation point and the other at the tip of the tab. However, the latter seems to be the stronger. The shear layer behind the tabbed cylinder is divided into two parts, one part upstream the tab and the other part downstream the tab, as shown in Figure 5(a). In general, the shear layer is convectively unstable [26]. Perturbations are introduced at the tip of the tabs in the shear layers, producing compression waves that travel upstream to the separation point, so that self-sustained oscillations can be achieved.

To understand the underlying self-sustained oscillations mechanisms in the shear layer shed from tabbed cylinder, 

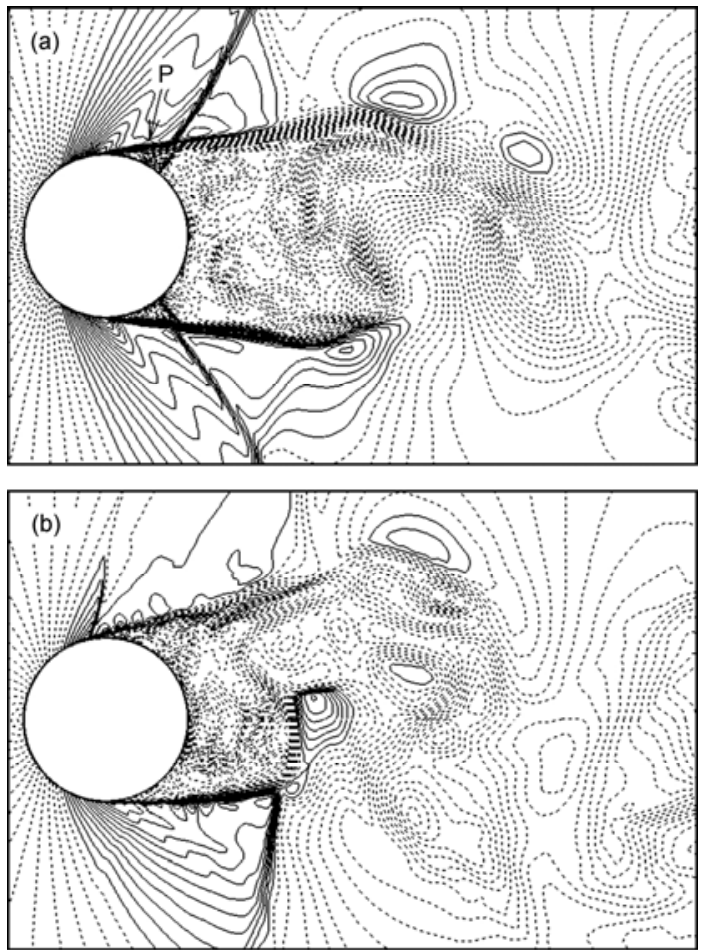

Figure 5 Instantaneous iso-contours of local Mach number $M_{l}$ in the mid-spanwise plane of the cylinder. (a) Tabbed cylinder; (b) circular cylinder. Here, solid lines denote $M_{l}>1$ and dashed lines $M_{l}<1$.

the pressure power spectral analysis is employed. Figure 6 shows the profile of pressure spectral density (PSD) at probe $\mathrm{P}$ marked in Figure 5(a). The profile of PSD contains a series of peaks at $S t=0.1,0.21,1.05$ and 1.12, which are associated with the resonant frequencies of compression waves in the shear layer and its harmonics. Based on the feedback model proposed by Rossiter [27], we may reasonably give a feedback model to describe one complete period of oscillation in the shear layer as

$$
T=L_{t} / U_{c}+L_{t} / a,
$$

where $L_{t}$ denotes the distance between the mean separation point and tip of the tab, $U_{c}$ the speed of upstream-propagating disturbances along the shear layer, and $a$ the local velocity of sound. Assuming $a$ as the free-stream velocity of sound $a_{\infty}$, the resonant Strouhal (St) numbers are predicted through

$$
S t=\frac{f D}{U_{\infty}}=\frac{D / L_{t}}{M_{\infty}+1 / K_{c}},
$$

where $f=1 / T$ denotes the resonant frequency, and $K_{c}$ is the velocity ratio $U_{c} / U_{\infty}$. According to the treatment [28], the value of $K_{c}$ equals 0.68 , approximately. We apply eq. (23) to obtain the non-dimensional frequency of the feedback loop $S t \approx 1$, which agrees well with the frequency value 1.05 and 1.12 issued from the spectral analysis in Figure 6. It means that the feedback model (23) may reliably predict the self-sustained oscillations in the shear layer. As Pastoor et al.

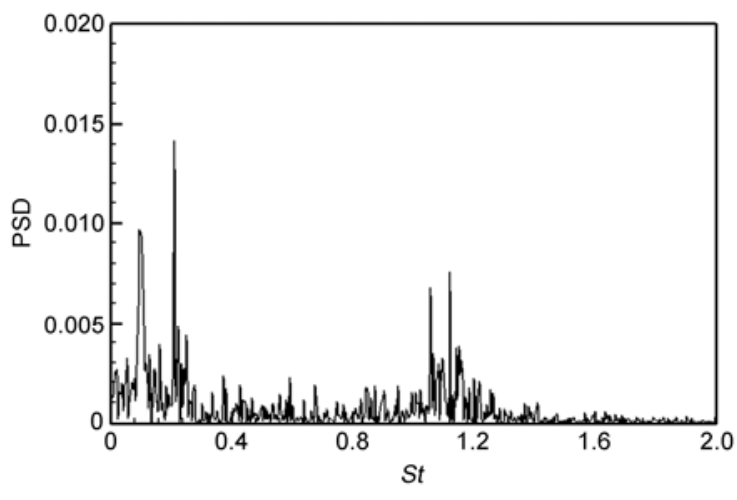

Figure 6 Profile of pressure power spectral density at the probe P.

[25] pointed out, evolution of large-scale vortex formations can be mitigated by high-frequency forcing. Thus, it is reasonable that vortex roll-up of the shear layer can be suppressed by these self-sustained oscillations.

\subsection{Characteristics of separated shear-layer evolution}

From the control perspective, in order to increase the base pressure and reduce the drag, the alternating character of the wake has to be mitigated or delayed [25]. The wake instability is closely associated with the shear-layer instability, and suppression of vortex roll-up inside the shear layer is enabler to increase the base pressure. Here, the mean behavior of separated shear-layer has been investigated to understand the shear layer growth with its evolution. Consequently, the vorticity thickness $\delta_{\omega}(x)$ has been used and can be describes as

$$
\delta_{\omega}(x)=\frac{\Delta \tilde{U}}{(\partial\langle\tilde{u}(x, z)\rangle / \partial z)_{\max }},
$$

where $\Delta \tilde{U}$ represents the resolved velocity difference magnitude across the shear layer. Figure 7(a) shows the evolution of $\delta_{\omega}$ along the shear layers shed from the cylinders. For the circular cylinder, the growth rate $\mathrm{d} \delta_{\omega} / \mathrm{d} x$ increase quickly, which is corresponding to the existence of shock/wake interaction, as typically shown in Figure 5(b). For the tabbed cylinder, shear layer can be divided into three parts: (i) upstream the tab $x / D<0.3$, the growth rate $\mathrm{d} \delta_{\omega} / \mathrm{d} x$ is nearly the same as that of circular cylinder and is equal to around 0.25 ; (ii) near the tab $0.3<x / D<0.6$, the growth rate $\mathrm{d} \delta_{\omega} / \mathrm{d} x$ is nearly zero, indicating that evolution of the shear layer has been suppressed by the tab; (iii) downstream the tab $x / D>0.6$, the shear layer develops linearly.

For the present compressible flow, the shear layer growth rate is affected by the compressibility effect which can be reasonably described by the convective Mach number $M_{c}$. $\delta_{\omega}$ decreases with $M_{c}$ for $M_{c}<1$ and does not change nearly when $M_{c}>1$. By means of the treatment [29], distributions of 

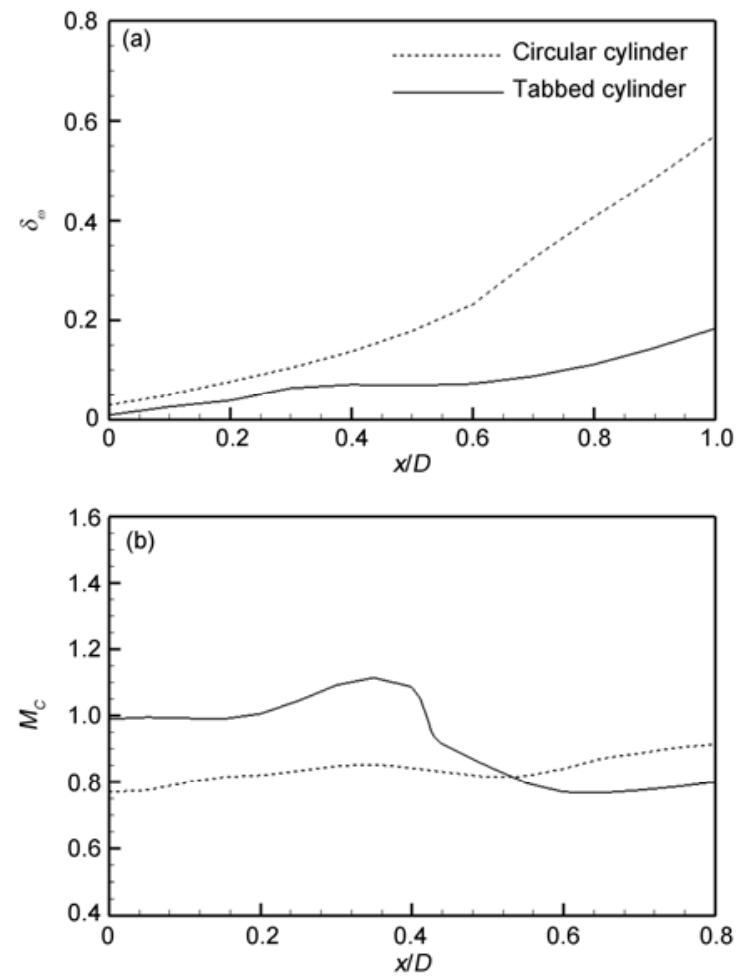

Figure 7 Evolution of flow field along the shear layers. (a) Vorticity thickness $\delta_{\omega} ;$ (b) convective Mach number $M_{c}$.

the convective Mach number $M_{c}$ are given in Figure 7(b). We have identified nearly $M_{c}>1$ in the initial stage of the shear layer for tabbed cylinder, which is corresponding to the self-sustained oscillations in the shear layer, while $M_{c}$ is around 0.8 in the initial part of the shear layer for circular cylinder. Thus, it is reasonable that the compressible effect lead to more stable shear layers of tabbed cylinder. In fact, the compressible effect is coupled to the self-sustained oscillations. Thus, suppression of the shear layer instability of tabbed cylinder is a result of their interaction.

Furthermore, the shear layer growth is coupled to the turbulent fluctuations [30]. Figure 8 shows the profiles of the local peak Reynolds stresses $\left\{u_{i}^{\prime \prime} u_{j}^{\prime \prime}\right\} / U_{\infty}^{2}$. For the shear layer shed from the tabbed cylinder, the values of the streamwise and transverse normal stresses and their shear stress remain small and increase very slowly. The turbulent fluctuations inside the shear layer shed from tabbed cylinder have been suppressed significantly, leading to the low growth rate in Figure 7(a). To characterize the turbulent fluctuations around the cylinder, iso-contours of the mean turbulent kinetic energy are shown in Figure 9 in the cross-section planes. It is obvious that the turbulent kinetic energy is negligibly small in the near wake behind the tabbed cylinder. Turbulent fluctuations around a body are associated with the fluctuating forces exerting on it. Thus, this feature is associated with the lower fluctuating surface pressure in Figure 4(b).

\section{Conclusions}

The compressible flow past a tabbed cylinder has been studied numerically by means of a LES technique for a free-stream Mach number $M_{\infty}=0.75$ and a Reynolds number based on the diameter $R e=2 \times 10^{5}$. For comparison, the compressible flow past a corresponding circular cylinder also has been calculated. Results show that the mean drag coefficient of the tabbed cylinder is less than that of a corresponding circular cylinder with a drag reduction 33\%. The lift coefficient fluctuation is greatly suppressed to be nearly zero. Moreover, drag reduction due to the shearing process prevails over that due to the compressing process in this flow.

The drag reduction of tabbed cylinder is closely associated with the higher base pressure, and lower lift fluctuations are related to suppression of the turbulent fluctuations behind the tabbed cylinder. The shear layer instability plays an important role in the base pressure distributions. To increase the base pressure, evolution of the shear layer must be suppressed. In this flow, both the compressible effect and high-frequency forcing issued from the self-sustained oscillations result in suppression of the shear layer instability of tabbed cylinder, which can reasonably be associated with drag reduction and suppression of lift fluctuations.
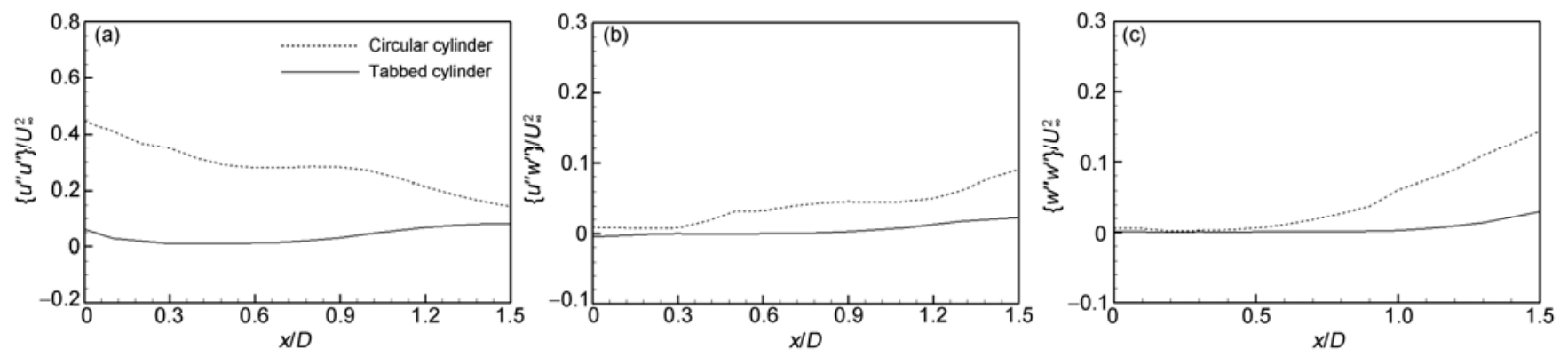

Figure 8 Evolution of local peak Reynolds stresses in the mid-spanwise plane of the cylinder. (a) Streamwise normal stress $\left\{u " u^{\prime \prime}\right\} / U_{\infty}^{2} ;(\mathrm{b})$ stream-transverse shear stress $\left\{u^{\prime \prime} w^{\prime \prime}\right\} / U_{\infty}^{2} ;$ (c) transverse normal stress $\left\{w^{\prime \prime} w^{\prime \prime}\right\} / U_{\infty}^{2}$. 

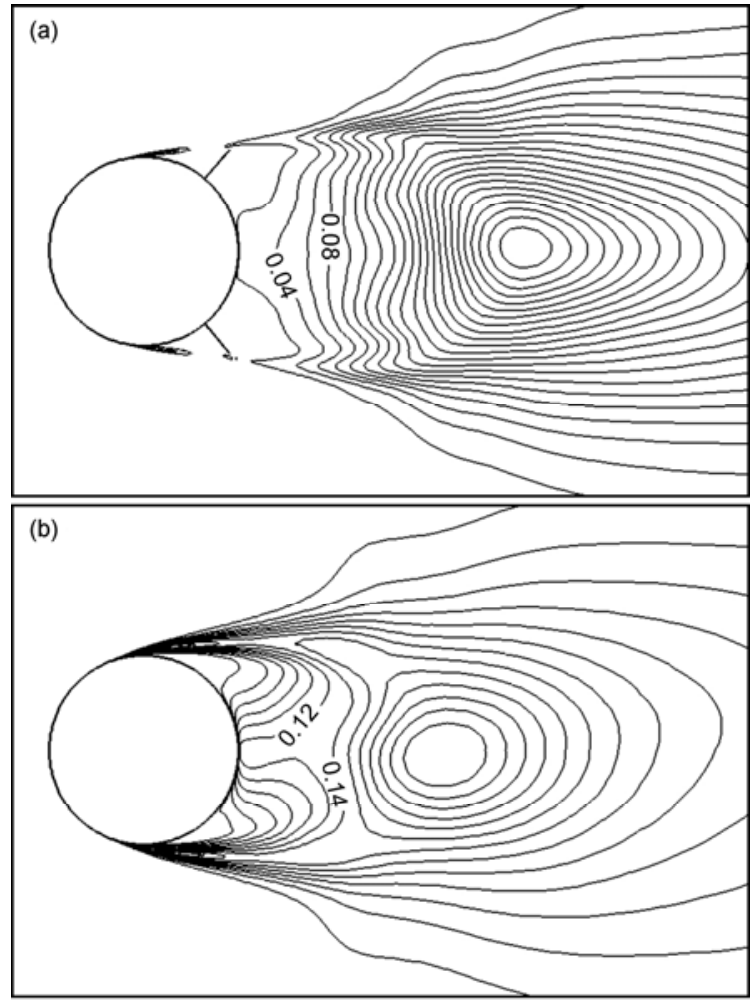

Figure 9 Iso-contours of mean turbulent kinetic energy in the crosssection planes. (a) Tabbed cylinder; (b) circular cylinder.

This work was supported by China Postdoctoral Science Foundation (20100481141 and 201104567), the Fundamental Research Funds for the Central Universities (NS2012032), Natural Science Foundation of Jiangsu Province (BK2011723), Jiangsu Planned Projects for Postdoctoral Research Funds (0902001C), Scientific Innovation Research of College Graduate in Jangsu Province (CX08B_035Z), and Innovation and Excellence Foundation of Doctoral Dissertation of NUAA (BCXJ08-01).

1 Choi H, Jeon W P, Kim J. Control of flow over a bluff body. Ann Rev Fluid Mech, 2008, 40: 113-139

2 Anderson E, Szewczyk A. Effects of a splitter plate on the near wake of a circular cylinder in 2- and 3-dimensional flow configurations. Exp Fluids, 1997, 23: 161-174

3 Hwang J Y, Yang K S, Sun S H. Reduction of flow-induced forces on a circular cylinder using a detached splitter plate. Phys Fluids, 2003, 15: 2433-2436

4 Akilli H, Karakus C, Akar A, et al. Control of vortex shedding of circular cylinder in shallow water flow using an attached splitter plate. J Fluid Eng, 2008, 130: 041401

5 Thombi L, Daisuke H, Yoshiaki N. Passive separation control on a body at transonic speed. Trans Japan Soc Aero Space Sci, 2002, 44: 229-237

6 Macha J M. Drag of circular cylinders at transonic Mach numbers. J Aircraft, 1977, 14: 605-607
7 Murthy V S, Rose W C. Detailed measurements on a circular cylinder in cross flow. AIAA J, 1978, 16: 549-550

8 Rodriguez $\mathrm{O}$. The circular cylinder in subsonic and transonic flow. AIAA J, 1984, 22: 1713-1718

9 Miserda R F B, Leal R G. Numerical simulation of the unsteady aerodynamic forces over a circular cylinder in transonic flow. AIAA Paper 2006-1408, 2006

10 Xu C Y, Chen L W, Lu X Y. Effect of Mach number on transonic flow past a circular cylinder. Chin Sci Bull, 2009, 54: 1886-1893

11 Xu C Y, Chen L W, Lu X Y. Large-eddy simulation of the compressible flow past a wavy cylinder. J Fluid Mech, 2010, 665: 238-273

12 Germano M, Piomelli U, Moin P, et al. A dynamic subgrid-scale eddy viscosity model. Phys Fluids A, 1991, 3: 1760-1765

13 Fureby C. On subgrid-scale modeling in large eddy simulations of compressible fluid flow. Phys Fluids, 1996, 8: 1301-1311

14 Salvetti M V, Banerjee S. A priori test of a new dynamic subgrid-scale model for finite difference large-eddy simulations. Phys Fluids, 1995, 7: 2831-2847

15 Desjardin P E, Frankel S H. Large-eddy simulation of a nonpremixed reacting jet: application and assessment of subgrid-scale combustion models. Phys Fluids, 1998, 10: 2298-2314

16 Moin P, Squires K, Cabot W, et al. A dynamic subgrid-scale model for compressible turbulence and scalar transport. Phys Fluids A, 1991, 3: $2746-2757$

17 Smagorinsky J. General circulation experiments with the privative equations. Part I. The basic experiment. Mon Weath Rev, 1963, 91: 99-164

18 Yoshizawa A. Statistical theory for compressible turbulent shear flows, with the application to subgrid modeling. Phys Fluids, 1986, 29: 2152-2164

19 Knight D, Zhou G, Okong'o N, et al. Compressible large eddy simulation using unstructured grids. AIAA Paper 1998-0535, 1998

20 Piomelli U. Large-eddy simulation: achievements and challenges. Prog Aerosp Sci, 1999, 35: 335-362

21 Martin M P, Piomelli U, Candler G V. Subgrid-scale models for compressible large-eddy simulations. Theor Comput Fluid Dyn, 1999 , 13: $361-376$

22 Hill D J, Pantano C, Pullin D I. Large-eddy simulation and multiscale modelling of a Richtmyer-Meshkov instability with reshock. J Fluid Mech, 2006, 557: 29-61

23 Wu J Z, Ma H Y, Zhou M D. Vorticity and Vortex Dynamics. Berlin: Springer Press, 2006

24 Wu J Z, Lu X Y, Zhuang L X. Integral force acting on a body due to local flow structures. J Fluid Mech, 2007, 576: 265-286

25 Pastoor M, Henning L, Noack B R, et al. Feedback shear layer control for bluff body drag reduction. J Fluid Mech, 2008, 608: 161-196

26 Oertel H. Wakes behind blunt bodies. Ann Rev Fluid Mech, 1990, 22 539-564

27 Rossiter J E. Wind-tunnel experiment on the flow over rectangular cavities at subsonic and transonic speeds. Royal Aircraft Establishment ARC R\&M No. 3438, 1964

28 Chen L W, Xu C Y, Lu X Y. Numerical investigation of the compressible flow past an aerofoil. J Fluid Mech, 2010, 643: 97-126

29 Simon F, Deck S, Guillen P, et al. Numerical simulation of the compressible mixing layer past an axisymmetric trailing edge. J Fluid Mech, 2007, 591: 215-253

30 Slessor M D, Zhuang M, Dimotakis P E. Turbulent shear-layer mixing: Growth-rate compressibility scaling. J Fluid Mech, 2000, 414: $35-45$

Open Access This article is distributed under the terms of the Creative Commons Attribution License which permits any use, distribution, and reproduction in any medium, provided the original author(s) and source are credited. 